\title{
Factors Associated with Attrition: Analysis of an HIV Clinic in Japan
}

\author{
Chieko Hashiba $^{1} \cdot$ Mayumi Imahashi $^{2} \mathbb{D} \cdot$ Junji Imamura $^{2,4} \cdot$ Masashi Nakahata $^{2} \cdot$ Ayumi Kogure $^{2} \cdot$ Hideto Takahashi $^{3}$. \\ Yoshiyuki Yokomaku
}

Published online: 18 February 2020

(c) The Author(s) 2020

\begin{abstract}
This study evaluated the impact of a public medical interpreter on the follow-up clinic attendance rate of foreign-born people with HIV who live in Japan. Participants were patients who visited Nagoya Medical Center from 2009 to 2016. Lost to follow-up was defined as an absence from follow-up visits for more than six months without any notification. A log-rank test was conducted to compare the lost-to-follow-up rates by patients' nation of origin and medical interpreter use. Of the 931 participants, 114 were foreign patients, whose overall attendance rate at 5 years was $75.5 \%$, which was significantly lower than that of Japanese patients $(94.1 \%, p<0.001)$. There was no significant difference in regular attendance with respect to medical interpreter use $(p=0.09)$. Social support in addition to a medical interpreter may be needed to improve attendance rates in the study population.
\end{abstract}

Keywords Medical interpreter $\cdot$ Language barrier $\cdot$ People living with HIV/AIDS · Health disparity

\section{Background}

The Japanese healthcare system has made a substantial contribution to promoting individuals' health in Japan. Nevertheless, people with limited Japanese proficiency, e.g., immigrants, may have limited access to appropriate health information or not receive the full benefits of the healthcare system due to their language barrier [1]. Notably, English is not often used, even in metropolitan cities in Japan. Thus far, no national-level law in Japan regarding the provision of medical interpreting services to patients with limited Japanese proficiency has been established, although some

Electronic supplementary material The online version of this article (https://doi.org/10.1007/s10903-020-00982-y) contains supplementary material, which is available to authorized users.

Mayumi Imahashi

mayumi.imahashi@nnh.go.jp

1 Department of Nursing, Nagoya Medical Center, 4-1-1 Sannomaru, Naka-ku, Nagoya 460-0001, Japan

2 Department of Infectious Diseases, Nagoya Medical Center, 4-1-1 Sannomaru, Naka-ku, Nagoya 460-0001, Japan

3 National Institute of Public Health, 2-3-6 Minami, Wako 351-0197, Japan

4 Department of Infectious Diseases, Sendai Medical Center, 2-8-8 Miyagino Miyagino-ku, Sendai 983-8520, Japan local authorities have introduced the provision of medical interpreters [2].

The difficulties of people living with HIV (PLWH) in Japan who have a language barrier experience difficulties, including connecting with healthcare facilities to maintain adequate drug adherence [3], dealing with public offices to receive health insurance, and finding an appropriate interpreter or one who can rectify the existing life-threatening interpretation errors that occur when ad hoc interpreters are used [4].

To reduce language barriers among PLWH in our clinic, in 2012, we introduced the "Aichi Medical Interpretation System" (AiMIS) [5]. This confidential service delivers medical interpreters to healthcare settings, arranges teleinterpreters, translates medical documents, and provides multilingual manuals for patient care. All medical interpreters in AiMIS are trained and certified before they are dispatched. Patients can choose an interpreter in person or over the phone. When patients request an attending medical interpreter for their appointments, a clinic clerk contacts AiMIS, and the cost of this service is met by the clinic.

Thus, this cross-sectional study was aimed to examine whether the use of a medical interpreter among PLWH with limited Japanese proficiency has a positive association on regular attendance to the HIV clinic. 
In this study, we (1) compared the rate of clinic attendance between foreign-born and Japanese patients, (2) compared the attendance rate among foreign-born patients based on AiMIS use, and (3) identified the determinants of regular follow-up visits.

We hypothesized that (1) Japanese patients would have a better attendance rate than would foreign-born patients, (2) AiMIS use would improve the attendance rates of foreignnational PLWH at our HIV clinic, and (3) the longer the foreign nationals live in Japan, the lower the rate of lost to follow-up.

\section{Methods}

\section{Participants}

Participants were recruited from an HIV specialty clinic at Nagoya Medical Center, Nagoya City, Aichi prefecture, and all patients in this clinic were HIV positive. The clinic has approximately $1300 \mathrm{HIV}$ patient visits per year.

The inclusion criteria for study participation were patients who visited our clinic initially between October 2009 and December 2016 and were at least 15 years old. The exclusion criteria were aged less than 15 years old and who had visited our clinic initially before October 2009 or after December 2016. One patient who grew up in Japan and was a native Japanese speaker was classified as a foreign-born participant because his nation of origin was not Japan.

We introduced a service in which we provided a medical interpreter to all the patients whose nation of origin was other than Japan. All medical interpreters were sent based on patient consent.

\section{Data Collection}

We analyzed demographic and clinical data recorded in the electronic medical records of patients first registered in our clinical database between October 2009 and December 2016. The requirement for written informed consent was waived. The opportunity to opt out of this study was made clear via an announcement in the clinic. Nurses in our clinic explained this study and the opportunity to opt out of this study to the patients after their appointments with doctors. For those with an insufficient understanding of Japanese, the nurses explained the study, using medical interpreters or healthcare workers who spoke English fluently.

\section{Measures}

Demographic variables included age, gender, and native language. Age was defined as the age when a patient was first registered in our database. Clinical and socioeconomic factors included years of residence in Japan, sexual orientation, whether living with family, health insurance, employment status, social welfare, disability certificates, treatment of HIV/AIDS, disease status at initial visit, and use of AiMIS. We also collected the number of times that AiMIS was used based on the communication records between AiMIS and our clinic when we requested interpreter services. The frequency of AiMIS use was recorded from May 2012 to August 2017. The lost-to-follow-up period was October 2009 to August 2017.

The primary efficacy analysis of AiMIS involved a comparison of the rate of foreign-born patients who visited our clinic regularly at successive points in time. Lost to followup was defined as not visiting our clinic for more than six months after the last visit without any notification. Therefore, we excluded patients from the lost-to-follow-up group if they returned to their home countries and notified our clinic. Treatment failure was defined as having an HIV-1 viral load of more than 200 copies $/ \mathrm{ml}$ [6].

\section{Analysis}

\section{Statistical Analysis}

Fisher's exact test was performed to compare the rates of certain factors (sex, sexual orientation, socioeconomic status at initial visit, AiMIS use, and distance from Nagoya Medical Center) between the lost-to-follow-up and non-lost-tofollow-up groups. To compare continuous variables, we used a Mann-Whitney $U$ test, as data on the patients' age and years of residence in Japan were not normally distributed. A $p$-value of less than 0.05 was considered statistically significant. Data were analyzed with STATA software, version 15.0 (College City, TX, USA). The distance from our clinic was measured by spatial analysis, which is described in the supplemental material.

The Kaplan-Meier method was used to compare the regular visit rate (1) between Japanese and foreign-born patients, (2) between patients who used AiMIS for their appointment and patients who did not, and (3) stratified by the frequency of AiMIS use. The log-rank test was used to examine the differences between the groups.

To analyze patients' demographics and socioeconomic status as determinants of regular attendance, we used the multivariate statistical model after adjusting for sex, sexual orientation (heterosexual vs. other), socioeconomic factors, AiMIS use, age ( $>35$ years vs. other), years of residence in Japan ( $>10$ years vs. other), and distance from the clinic ( $>17 \mathrm{~km}$ vs. other). We conducted a univariate analysis for each factor and then chose the factors based on a clinical perspective. Finally, we selected a model of multivariate analysis and conducted a multivariate analysis. 
The Institutional Review Board of Nagoya Medical Center approved the protocol and use of clinical data for this study (\#2017-89).

\section{Results}

\section{Patients' Demographics, Socioeconomic Status, and Clinical Data}

Of the 931 registered patients, 114 were foreign-born; among them, 44 patients were from Brazil, followed by 11 from Peru and 8 from Indonesia (Fig. 1). A total of 20 patients became lost to follow-up. Patients' demographics and socioeconomic status by lost-to-follow-up status are presented in Table 1.

The foreign patients' median age at initial visits was 36 years for those non-lost-to-follow-up and 32 years for those lost to follow-up ( $p=0.052)$. The median years of residence in Japan was 10 years for both groups $(p=0.49)$. The proportion of men was $76.6 \%$ in the non-lost-to-follow-up group and $85.0 \%$ in the lost-to-follow-up group $(p=0.41)$. A total of $58.6 \%$ of patients in the non-lost-to-follow-up group and $45.0 \%$ in the lost-to-follow-up group were heterosexual $(p=0.47)$. At the initial visit, $50.0 \%$ of patients in the lostto-follow-up group and $60.6 \%$ of patients in the non-lost-tofollow-up group were living with their families $(p=0.91)$. Of patients in the non-lost-to-follow-up group, $90.4 \%$ have health insurance as compared to $65.0 \%$ in the lost-to-follow-up group, indicating a significant difference $(p=0.01)$. A total of $61.7 \%$ of patients in the non-lost-to-follow-up group and $55.0 \%$ in lost-to-follow-up group were employed $(p=0.35)$. At the initial visit, anti-retroviral therapy (ART) was introduced to $31.9 \%$ of patients in the non-lost-to-follow-up group and to $25.0 \%$ of patients in lost-to-follow-up group $(p=0.54)$. Further, $35.1 \%$ of non-lost-to-follow-up group patients and $20.0 \%$ of lost-to-follow-up group had AIDS $(p=0.36)$.

The Japanese patients' median age at initial visits was 38 years. Men comprise $96.3 \%$ of the sample, and women, $3.7 \%$. A total of $18.6 \%$ of Japanese patients were heterosexual. At the initial visit, $54.4 \%$ of Japanese patients were living with their families. Of the Japanese patients, $96.0 \%$ had health insurance and $76.1 \%$ were employed. At the initial visit, ART was introduced to $16.9 \%$ of the Japanese patients, and $35.4 \%$ of the Japanese patients had AIDS.

\section{AiMIS Use}

Among the 114 foreign-born patients, 49 (43.0\%) used AiMIS from the start of this service to August 2017. Among the 49 AiMIS users, Portuguese was the language most required for interpreters $(n=30,61.2 \%)$, followed by Spanish $(n=8,16.3 \%)$ and English $(n=6,12.2 \%)$. Patients' primary native languages are shown in Supplement 3.

The median number of times AiMIS was used by each patient was five (interquartile range $[\mathrm{IQR}]=1-11$ ). Among the 49 patients who utilized AiMIS, 13 (26.5\%) used it only once.

Among the 90 patients who were followed up at more than 365 days, 22 patients showed treatment failure. There was no significant difference between patients with and without treatment failure with respect to AiMIS $(p=0.25)$ (Supplement 4).

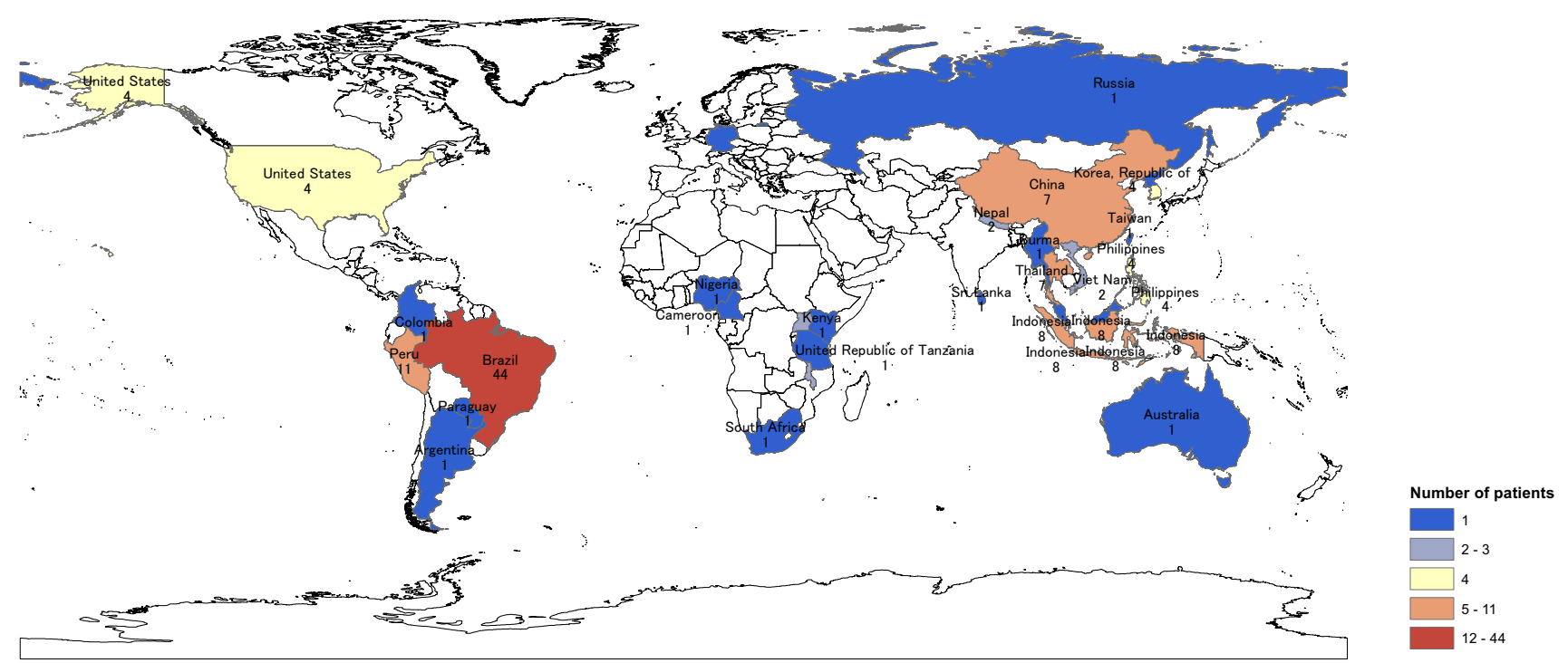

Fig. 1 Map of the home countries of the foreign patients in the study sample. Each number represents the number of patients from the country 
Table 1 Sociodemographic characteristics of the participants

\begin{tabular}{|c|c|c|c|c|}
\hline & \multicolumn{2}{|l|}{ Foreign patients } & \multirow[t]{3}{*}{$p$} & \multirow{3}{*}{$\begin{array}{l}\text { Japanese patients } \\
\mathrm{n}=817\end{array}$} \\
\hline & \multicolumn{2}{|l|}{ Lost to follow-up } & & \\
\hline & Yes $(n=20)$ & No $(n=94)$ & & \\
\hline Age at initial visit, median [IQR] & 32 [29-37] & $36[31-47]$ & 0.052 & $38[30.9-46.3]$ \\
\hline Years of residence in Japan, median [IQR] & $10[3-19]$ & $10[5-20]$ & 0.49 & NA \\
\hline \multicolumn{5}{|l|}{ Sex, n $(\%)$} \\
\hline Male & $17(85.0)$ & $72(76.6)$ & 0.41 & $787(96.3)$ \\
\hline Female & $3(15.0)$ & $22(23.4)$ & & $30(3.7)$ \\
\hline \multicolumn{5}{|l|}{ Sexual orientation, $\mathrm{n}(\%)$} \\
\hline Homosexual & $5(25.0)$ & $22(23.6)$ & 0.47 & $377(46.2)$ \\
\hline Bisexual & $5(25.0)$ & $12(12.9)$ & & $257(31.5)$ \\
\hline Heterosexual & $9(45.0)$ & $54(58.6)$ & & $152(18.6)$ \\
\hline Other/unknown & $1(5.0)$ & $5(5.4)$ & & $30(3.7)$ \\
\hline \multicolumn{5}{|l|}{ At initial visit, $\mathrm{n}(\%)$} \\
\hline Living with family & $12(60.0)$ & $57(60.6)$ & 0.91 & $444(54.4)$ \\
\hline Insured & $13(65.0)$ & $85(90.4)$ & 0.01 & $784(96.0)$ \\
\hline Employed & $11(55.0)$ & $58(61.7)$ & 0.35 & $622(76.1)$ \\
\hline Receiving social welfare & $1(5.0)$ & $7(7.5)$ & 0.2 & $411(5.0)$ \\
\hline Receiving disability certificates & $4(20.0)$ & $20(21.3)$ & 0.9 & $120(14.7)$ \\
\hline Receiving ART & $5(25.0)$ & $30(31.9)$ & 0.54 & $138(16.9)$ \\
\hline AIDS & $5(20.0)$ & $35(35.1)$ & 0.36 & $289(35.4)$ \\
\hline \multicolumn{5}{|l|}{ AiMIS use n (\%) } \\
\hline Once or more & $11(55.0)$ & $38(40.4)$ & 0.23 & NA \\
\hline Distance $(\mathrm{km})$ from NMC, median [IQR] & $17.6[3.2-32.0]$ & $16.6[7.6-31.8]$ & 0.52 & NA \\
\hline
\end{tabular}

$I Q R$ interquartile range, $A R T$ anti-retroviral therapy, $A I D S$ acquired immuno-deficiency syndrome, AiMIS Aichi Medical Interpretation System, NMC Nagoya Medical Center, $N A$ not applicable

\section{Attendance Rate}

The median follow-up periods among Japanese and foreign-born patients were 1255 days $(\mathrm{IQR}=588-1972)$ and 809.5 days ( $\mathrm{IQR}=460-1441$ ), respectively.

The attendance rate of Japanese patients in our clinic was $98.3 \%, 97.4 \%, 96.8 \%, 95.8 \%$, and $94.1 \%$ at Years $1,2,3,4$, and 5 , respectively. In contrast, the attendance rate of the foreign-born patients after initial visits was $95.1 \%, 86.0 \%$, $83.1 \%, 75.5 \%$, and $75.5 \%$ at Years $1,2,3,4$, and 5, respectively (Fig. 2). The log-rank test indicated that there was a statistically significant difference between Japanese patients' attendance rate and that of foreign-born patients $(p<0.01)$.

Stratified by the use of AiMIS, the rate of attendance appeared to be higher in the absence of AiMIS use; however, the difference was not significant ( $p=0.09$ ) (Fig. 3). The rate of attendance seemed to be higher among individuals who used AiMIS more than five times during their follow-up period days until day 1362. The log-rank test, however, showed no significant difference in the attendance rate $(p=0.69)$ (Fig. 3). The attendance rate dropped dramatically after 1362 days. One patient terminated his attendance because he started work outside of the Aichi prefecture, and another did so due to financial problems.

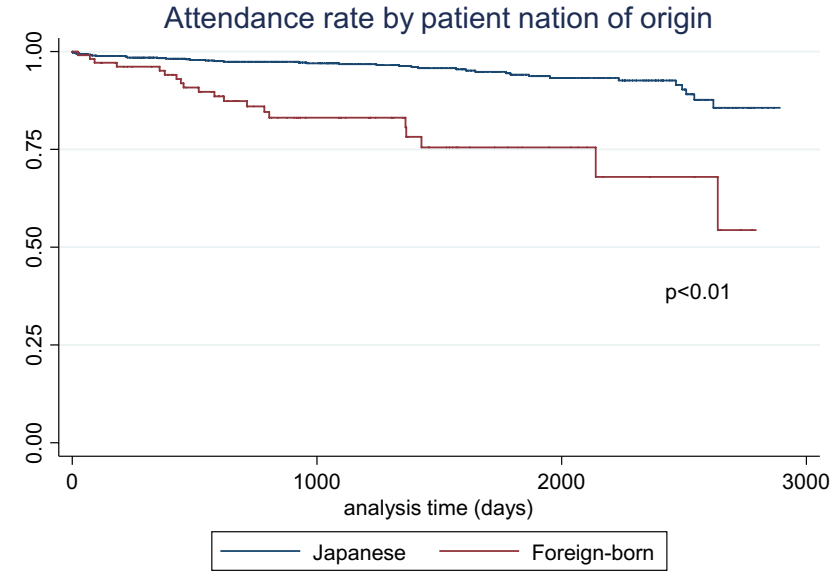

Fig. 2 Attendance rate by patient nation of origin

\section{Determinants of Attendance}

Based on the univariate analysis, no factor was related to lost to follow-up (Table 2). We excluded the factor of "Employed" and "Receiving ART" from the multivariate analysis, as the factor "Employed" was strongly correlated to the factor "Receiving social welfare," and the 
Fig. 3 Attendance rate by patients' AiMIS use and the number of times they used AiMIS

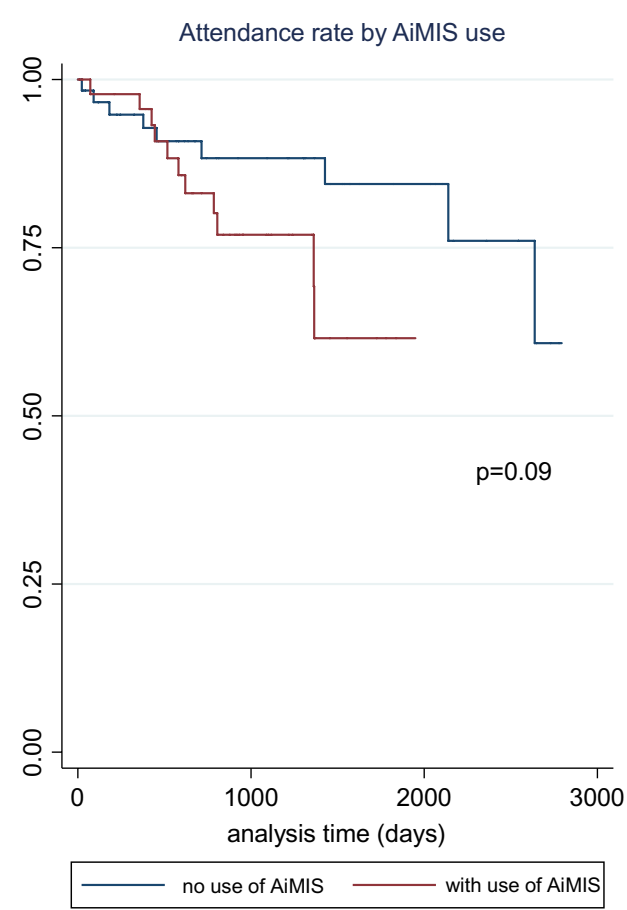

Attendance rate by the number of AiMIS use

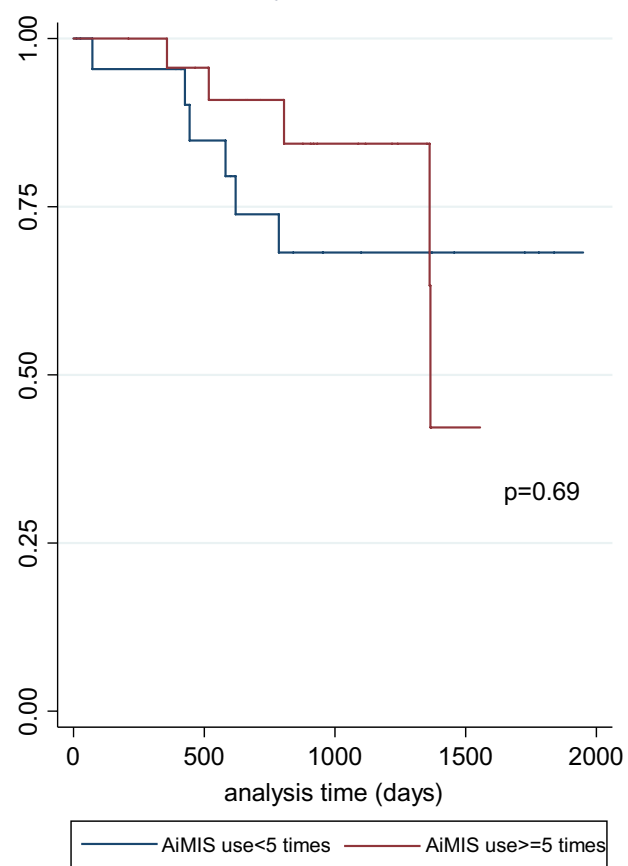

factor "Receiving ART" also was strongly correlated to the factor "Receiving disability certificates" from a clinical perspective.

Based on the multivariate analysis, the risk of lost to follow-up for males (odds ratio 1.71; 95\% CI [0.27, 10.90]; $p=0.57$ ) and heterosexual patients (odds ratio $0.65 ; 95 \% \mathrm{CI}$ $[0.18,2.26] ; p=0.50)$ did not differ significantly from that of their counterparts (Table 2). Among the socioeconomic factors, no factor was significantly associated with attendance. Insurance was not significantly associated with attendance, after adjusting for patients' demographics, other socioeconomic factors, and clinical status.

\section{Discussion}

The results showed a significant difference in the attendance rates between Japanese and foreign-born patients. The use of medical interpreters, however, did not significantly affect attendance in this study population.
Table 2 Patient characteristics associated with lost to follow-up

\begin{tabular}{lll}
\hline & $\begin{array}{l}\text { Univariate analysis } \\
\text { Odds ratio [95\% confidence } \\
\text { interval] }\end{array}$ & $\begin{array}{l}\text { Multivariate analysis } \\
\text { Odds ratio [95\% } \\
\text { confidence interval] }\end{array}$ \\
\hline Sex (male) & $1.73[0.46-6.46]$ & $1.71[0.27-10.90]$ \\
Heterosexual & $0.59[0.22-1.56]$ & $0.65[0.18-2.26]$ \\
Living with family & $0.7[0.33-1.50]$ & $0.65[0.26-1.63]$ \\
AIDS at initial visits & $1.12[0.57-2.20]$ & $0.53[0.15-1.87]$ \\
Employed & $1.22[0.61-2.41]$ & \\
Insured & $0.58[0.20-1.74]$ & $0.69[0.27-1.77]$ \\
Receiving social welfare & $1.89[0.75-4.75]$ & $4.7[1.00-22.08]$ \\
Receiving disability certificate & $0.93[0.28-3.08]$ & $1.75[0.44-6.95]$ \\
Receiving ART & $0.71[0.24-2.14]$ & \\
AiMIS use & $1.8[0.68-4.76]$ & $2.19[0.66-7.22]$ \\
Age at initial visit $>35$ years old & $0.69[0.26-1.82]$ & $0.55[0.17-1.73]$ \\
$>10$ years of residence in Japan & $1.36[0.50-3.68]$ & $1.83[0.57-5.86]$ \\
Living $>17$ km away from NMC & $1.11[0.41-3.00]$ & $1.3[0.39-4.29]$ \\
\hline
\end{tabular}

NMC Nagoya Medical Center 
Our data showed that the attendance rate of the foreignborn population in our HIV clinic at 5 years was $75.5 \%$, which was lower than that of the Japanese patients at that point in time (94.1\%). This rate, however, was not necessarily low as compared with other settings. For example, Diaz et al. reported that $13.7 \%$ of HIV patients did not attend medical appointments regularly [7]. They also mentioned that one of the factors related to non-regular visit was birthplace, such as Sub-Saharan Africa or Latin-America, indicating that differences between languages and cultures are barriers for patients to engage in regular attendance. In Nepal, only $32.6 \%$ of HIV-positive individuals were found to regularly visit a clinic [8]. Regular attendance was promoted by family support, participation in support programs, and knowledge of ART benefits. Regular attendance was hindered, however, by more extended commutes to the clinic and self-rated health status of very good, good, or fair. In our study, AIDS status at the initial visit was not associated with regular attendance. Moreover, the distribution of foreign-born patients' residences was not different between the non-regular and regular attendance groups. This result may be due to the developed Japanese infrastructure, which facilitates patients visits to hospitals across long distances.

Although we hypothesized that the use of AiMIS would have a positive impact on the attendance of foreign-born PLWH to our clinic, the attendance rates did not show a significant difference when compared by AiMIS use and number of times that AiMIS was used. Therefore, it was assumed that foreign-born patients who did not use AiMIS (1) were able to speak Japanese without an interpreter, (2) had disclosed their HIV infection to family or friends who were able to act as interpreters, or (3) refused to use a medical interpreter because they were afraid that the interpreter could breach confidentiality. One study reported that the disclosure rate of HIV was explicitly lower than that of other chronic diseases, such as viral hepatitis or diabetes mellitus [9]. Therefore, the impact of medical interpreters for patients of other chronic diseases might be more significant than for HIV patients. It also can be assumed that patients who used AiMIS fewer than five times were required to have a medical interpreter based not on their request but on that of the healthcare worker. That is, healthcare workers thought that the patients were not proficient enough in Japanese.

The impact of the use of medical interpreters on HIV-1 treatment was not significant. In regard to this finding, we were able to conclude only that the language barrier was not the only reason for the lost-to-follow-up status among foreign born patients. There might be some other reasons that limited their attendance.

Patient factors did not have an impact on being lost to follow-up when adjusted by multivariate analysis. Nevertheless, "Receiving social welfare" was a significant factor; if the number of participants had been larger, it may have been a factor that had an impact on being lost to follow-up. Thus, further study is warranted.

Our study has some limitations. First, the number of foreign-born nationals was small. Therefore, the lost to followup in terms of attendance strongly affected the attendance rate curve when analyzing the impact of AiMIS use. Second, it is impossible to ascertain the reasons that the patients who were lost to follow-up terminated their visits. Thus, we had to assume the reasons for non-attendance. If we could identify the reasons, we would offer assistance with patients' issues so that they could remain connected to healthcare. Third, our study sample was geographically limited and had different racial characteristics from those of Japan as a whole. The Aichi prefecture, where our facility is located, has approximately 200,000 foreign-born inhabitants, which is the second largest foreign-born population in Japan [10]. Due to manufacturing industries, nearly half work for the factories related to these sectors [11]. A majority are from Brazil, although Chinese are the largest group of residents from foreign nations in Japan [10]. Therefore, if the same analyses were conducted in a different area, the results might differ. Fourth, we analyzed patients who initially visited our clinic from 2009 to 2016, although the AiMIS service did not start until 2012. To minimize selection bias both for foreign-born and Japanese patients, we conducted an analysis that began with 2009, when electronic medical records were introduced into our clinic. Finally, we did not evaluate each patient's Japanese proficiency objectively. Instead, a subjective assessment of patients' Japanese speaking ability was made by nurses in our clinic (Supplement 5). According to the data, there was not a significant difference in the Japanese speaking ability between the lost-to-follow-up and non-lost-to-follow-up groups $(p=0.70)$. In the analysis of determinants of attendance, the data were not included due to not being measured objectively. Thus, we are not able to exclude the possibility that there might be a significant difference in the rate of regular attendance according to AiMIS use if we excluded from the analysis the patients who could speak Japanese very well.

Thus, we can conclude that the intervention of medical interpreters did not improve the rate of attendance to our clinic. Hence, to improve the attendance rate of foreign-born patients, not only medical interpreter services, but also comprehensive social support and an employment environment that facilitates receiving healthcare are needed. That is, in Japan, services provided by AiMIS should be combined with administrative and social services. 


\section{Contribution to the Literature}

To our knowledge, this study is the first to examine the regular attendance rate of PLWH in Japan, using Kaplan-Meier curves, tracking each patient's attendance at follow-up visits. This analysis demonstrated significantly different attendance rates between Japanese and foreign-born patients. Further, the use of medical interpreters alone in the clinical setting did not improve the regular attendance rates of foreign-born HIV patients, suggesting that it is necessary to provide not only language support but also comprehensive social support for foreign-born HIV patients.

Acknowledgements This study was funded by the Health, Labour and Welfare Sciences Research Grants (Research on HIV/AIDS), Ministry of Health, Labour and Welfare of Japan (H29-AIDS-ippan-004) and Health, Labour and Welfare Policy Research Grants (Research on HIV/AIDS), Ministry of Health, Labour and Welfare of Japan (H29-AIDS-shitei-001)

\section{Compliance with Ethical Standards}

Conflict of interest The authors declare that there is no conflict of interest.

Ethical Approval The Institutional Review Board of Nagoya Medical Center approved the protocol and use of clinical data for this study (\#2017-89). The requirement for written informed consent was waived. The opportunity to opt out of this study was made clear by an announcement in the clinic.

Open Access This article is licensed under a Creative Commons Attribution 4.0 International License, which permits use, sharing, adaptation, distribution and reproduction in any medium or format, as long as you give appropriate credit to the original author(s) and the source, provide a link to the Creative Commons licence, and indicate if changes were made. The images or other third party material in this article are included in the article's Creative Commons licence, unless indicated otherwise in a credit line to the material. If material is not included in the article's Creative Commons licence and your intended use is not permitted by statutory regulation or exceeds the permitted use, you will need to obtain permission directly from the copyright holder. To view a copy of this licence, visit http://creativecommons.org/licenses/by/4.0/.
2. Yoshitomi S. The importance of medical interpreters in local medical institutions: from the establishment of a medical interpreting system in hyogo prefecture. Migr Policy Rev. 2009;1:140-51.

3. Nakagawa F, Lodwick RK, Smith CJ, Smith R, Cambiano V, Lundgren JD, Delpech V, Phillips AN. Projected life expectancy of people with hiv according to timing of diagnosis. AIDS. 2012;26:335-43.

4. Flores G, Laws MB, Mayo SJ, Zuckerman B, Abreu M, Medina L, Hardt EJ. Errors in medical interpretation and their potential clinical consequences in pediatric encounters. Pediatrics. 2003;111:6-14.

5. Aichi Medical Interpretations System. Aichi medical interpretations system, information. 2012. https://www.aichi-iryou-tsuya ku-system.com/pt/pte.html. Accessed 30 July 2018.

6. Saag MS, Benson CA, Gandhi RT, Hoy JF, Landovitz RJ, Mugavero MJ, Sax PE, Smith DM, Thompson MA, Buchbinder SP, Del Rio C, Eron JJ Jr, Fatkenheuer G, Gunthard HF, Molina JM, Jacobsen DM, Volberding PA. Antiretroviral drugs for treatment and prevention of hiv infection in adults: 2018 recommendations of the international antiviral society-USA panel. JAMA. 2018;320:379-96.

7. Diaz A, Tenwg A, Marcos H, Gutierrez G, Gonzalez-Garcia J, Moreno S, Barrios AM, Arponen S, Portillo A, Serrano R, Garcia MT, Perez JL, Toledo J, Royo MC, Gonzalez G, Izquierdo A, Viloria LJ, Lopez I, Elizalde L, Martinez E, Castrillejo D, Aranguren R, Redondo C, Diez M, Hospitalaria E. Factors determining irregular attendance to follow-up visits among human immunodeficiency virus patients: Results of the hospital survey of patients infected with human immunodeficiency virus. Enferm Infecc Microbiol Clin. 2015;33:324-30.

8. Ayer R, Kikuchi K, Ghimire M, Shibanuma A, Pant MR, Poudel $\mathrm{KC}$, Jimba M. Clinic attendance for antiretroviral pills pick-up among HIV-positive people in Nepal: roles of perceived family support and associated factors. PLoS ONE. 2016;11:e0159382.

9. Kittner JM, Brokamp F, Jager B, Wulff W, Schwandt B, Jasinski J, Wedemeyer H, Schmidt RE, Schattenberg JM, Galle PR, Schuchmann M. Disclosure behaviour and experienced reactions in patients with hiv versus chronic viral hepatitis or diabetes mellitus in germany. AIDS Care. 2013;25:1259-70.

10. Ministry of Justice. The number of foreigners residing in Japan, 2017. 2018. https://www.moj.go.jp/nyuukokukanri/kouhou/nyuuk okukanri04_00073.html. Accessed 1 Sept 2018.

11. Aichi Labour Bureau. The number of places of business employ foreign national and workers by industry. 2016. https://jsite.mhlw. go.jp/aichi-roudoukyoku/var/rev0/0117/7146/201712611281.pdf. Accessed 1 Sept 2018.

Publisher's Note Springer Nature remains neutral with regard to jurisdictional claims in published maps and institutional affiliations.

\section{References}

1. Kreps GL, Sparks L. Meeting the health literacy needs of immigrant populations. Patient Educ Couns. 2008;71:328-32. 